\title{
Interrelation between donor and recipient heart rates during exercise after heterotopic cardiac transplantation
}

\author{
SALIM YUSUF,^ ANDREW MITCHELL, MAGDI H YACOUB \\ From Harefield Hospital, Harefield, Middlesex
}

SUMMARY The interrelation between the rates of the innervated recipient heart and the denervated donor heart at rest, on standing, and during the different phases of maximal exercise was studied in nine patients 1-6 months after heterotopic cardiac transplantation. The resting heart rate was significantly higher in the donor heart compared with the recipient heart. Eight of the nine recipient hearts and none of the donor hearts showed an increase in heart rate on standing up. All patients were exercised using a Bruce protocol until fatigued. The increase in heart rate during the first three minutes of exercise was lower in the donor hearts $(10.7(6)$ beats $/ \mathrm{min})$ than in the recipient hearts (30(4.8) beats/min) but the peak heart rates were almost identical (donor hearts $152.7(2.9)$ and recipient hearts $152(2.8)$ beats/min). Five of the nine donor hearts and none of the recipient hearts showed a significant increase in heart rate after cessation of exercise. Three minutes after exercise heart rate had decreased by only $0.2(5.7)$ beats/min in the donor hearts compared with $33.6(6)$ beats/min in the recipient hearts. In spite of these differences in response between the donor hearts and recipient hearts, there were significant correlations between the two heart rates in the same patient at rest, after the first three minutes of exercise, at peak exercise, and during recovery.

Heterotopic cardiac transplantation presents a unique opportunity to compare the responses of the innervated recipient heart and the denervated donor heart simultaneously to identical stimuli. Although there have been previous reports of changes in heart rate in the denervated donor heart to exercise in experimental $^{1}$ and clinical ${ }^{2}$ orthotopic cardiac transplantation, there are no reports of similar studies after heterotopic cardiac transplantation.

The purpose of this study was to examine changes in heart rates of the donor and recipient hearts and their interactions during changes in posture and dynamic exercise. Such studies could be of value in clarifying the role of the nervous system in regulating heart rate during different phases of exercise and in understanding the cardiovascular dynamics of patients after heterotopic transplantation.

Requests for reprints to Mr Magdi H Yacoub, FRCS, Harefield Hospital, Harefield, Middlesex UB9 6JH.

*Present address: Clinical Trials Branch, NHLBI, Federal Building, Wisconsin Avenue, Bethesda, MD 20205, USA.

Accepted for publication 22 March 1985

\section{Patients and methods}

Nine patients who had undergone heterotopic cardiac transplantation 1-6 months earlier were studied. The Table outlines the clinical characteristics of these patients, details of the underlying cardiac pathology of the recipient heart, data regarding the donor heart and drug treatment, and the results of the most recent cardiac biopsy (of the donor heart).

None of these patients was receiving beta blockers, digitalis, or other cardiac drugs, nor did they have diabetes or other diseases that could affect the autonomic nervous system. One patient was studied one month after operation, two three months, and the rest six months. In all patients both the donor and recipient hearts were in sinus rhythm.

\section{OPERATIVE TECHNIQUE}

Heterotopic cardiac transplantation involves the insertion of the donor heart into the right chest in parallel with the recipient heart. Anastomoses are carried out between the donor and recipient superior vena cavae, left atria, aorta, and pulmonary arteries. 
Table Details of patients

\begin{tabular}{|c|c|c|c|c|c|c|}
\hline Case No & $\begin{array}{l}\text { Sexlage of recipient } \\
(y r)\end{array}$ & $\begin{array}{l}\text { Age of } \\
\text { donor }(y r)\end{array}$ & $\begin{array}{l}\text { Interval from operation } \\
\text { to exercise (month) }\end{array}$ & $\begin{array}{l}\text { Underlying } \\
\text { pathology }\end{array}$ & Drugs & $\begin{array}{l}\text { Recent biopsy } \\
\text { findings }\end{array}$ \\
\hline $\begin{array}{l}1 \\
2\end{array}$ & $\begin{array}{l}M / 27 \\
M / 42\end{array}$ & $\begin{array}{l}16 \\
14\end{array}$ & $\begin{array}{l}2 \\
5\end{array}$ & $\begin{array}{l}\text { CCM } \\
\text { IHD }\end{array}$ & $\begin{array}{l}\text { Imuran, cyclosporin A } \\
\text { Prednisolone, imuran, } \\
\text { cyclosporin A }\end{array}$ & $\begin{array}{l}\text { No rejection } \\
\text { No rejection }\end{array}$ \\
\hline 3 & $M / 46$ & 16 & 4 & IHD & $\begin{array}{l}\text { Prednisolone, imuran, } \\
\text { cyclosporin A }\end{array}$ & Mild rejection \\
\hline 4 & $\mathbf{M} / 50$ & 30 & 6 & IHD & $\begin{array}{l}\text { Prednisolone, imuran, } \\
\text { cyclosporin A }\end{array}$ & No rejection \\
\hline 5 & $M / 42$ & 19 & 5 & $\mathrm{CCM}$ & $\begin{array}{l}\text { Prednisolone, imuran, } \\
\text { cyclosporin A }\end{array}$ & Mild rejection \\
\hline $\begin{array}{l}6 \\
7 \\
8\end{array}$ & $\begin{array}{l}M / 45 \\
M / 59 \\
F / 30\end{array}$ & $\begin{array}{l}32 \\
36 \\
13\end{array}$ & $\begin{array}{l}2 \cdot 5 \\
3 \\
6\end{array}$ & $\begin{array}{l}\text { IHD } \\
\text { IHD } \\
\text { CCM }\end{array}$ & $\begin{array}{l}\text { Imuran, prednisolone } \\
\text { Imuran, prednisolone } \\
\text { Prednisolone, imuran, } \\
\text { cyclosporin A }\end{array}$ & $\begin{array}{l}\text { Mild rejection } \\
\text { No rejection } \\
\text { No rejection }\end{array}$ \\
\hline 9 & $M / 42$ & 20 & 1 & IHD & $\begin{array}{l}\text { Imuran, cyclosporin } \mathrm{A}, \\
\text { diuretics }\end{array}$ & No rejection \\
\hline
\end{tabular}

CCM, congestive cardiomyopathy; IHD, ischaemic heart disease.

This modified technique, where the vena cavae are anastomosed instead of the right atria, ${ }^{3}$ preserves the arteries to both sinoatrial nodes and the nerves to the recipient heart. In addition, aneurysmectomy with coronary artery bypass grafts to the recipient hearts were performed in six patients who had ischaemic heart disease (Table).

\section{STUDY PROTOCOL}

A modified 12 lead electrocardiogram was obtained at $25 \mathrm{~mm} / \mathrm{s}$ before the start of exercise with lead V6R recorded instead of V2. The QRS complex from the donor heart produced the dominant positive vector in V6R whereas that fróm the recipient heart produced a dominant positive vector in V5 (Fig. 1). In addition, the configuration of the QRS complexes was compared with the preoperative electrocardiogram to help distinguish the complexes from the donor and recipient hearts in the different leads.

Baseline recordings were obtained after at least 10 minutes in the sitting position and after $\mathbf{3 0}$ seconds of standing. Exercise was then started on a computerised treadmill (Marquette Case) using the Bruce protocol. ${ }^{4}$ A three lead electrocardiogram was printed out in the first five seconds of each minute of exercise and at every minute of recovery for at least 12 minutes. The leads monitored were those that enabled clear separation of the donor and recipient QRS complexes and usually included V1, V6R, and another lead. Figure 1 shows recordings in a patient at rest and at peak exercise. Heart rate was calculated from the mean of at least three consecutive $R R$ intervals for each heart at rest, at every minute of exercise, at peak exercise, and at every minute of recovery for at least 12 minutes.

\section{CONTROL SUBJECTS}

Eight healthy age matched male volunteers served as control subjects and followed the above protocol.

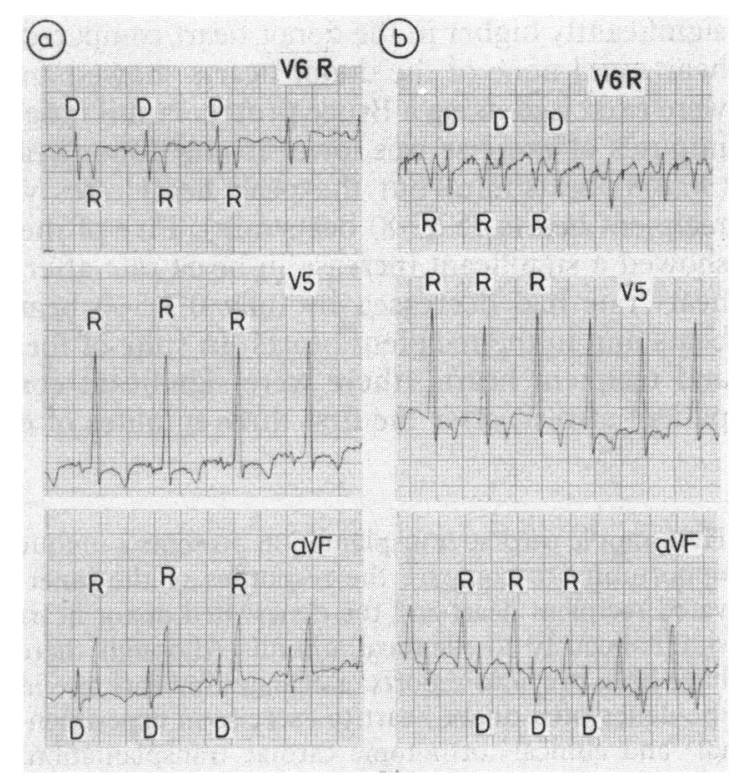

Fig. 1 Electrocardiograms from a patient (a) at rest and (b) at peak exercise showing separate identification of donor and recipient QRS complexes.

Their heart rates were recorded at rest, on standing, and at every minute of exercise, and during recovery for 12 minutes.

\section{STATISTICAL TECHNIQUES}

Standard paired $t$ tests, $\chi^{2}$ tests, and correlations were used to compare changes between the two heart rates. Results are expressed as mean (one standard error of mean). 
Results

\section{RESTING HEART RATES}

The resting heart rate was significantly higher in the donor hearts $(106(2)$ beats $/ \mathrm{min})$ than in their respective recipient hearts $(95(6)$ beats/min; $p<0.05$ ) (Figs. 2a and 3a). The donor heart rate was lower than the recipient heart rate in only one patient. There was a significant positive correlation between the two resting heart rates (Fig. 3$)(r=0.84 ; p<0.01)$. The resting heart rates of both the donor and recipient hearts were independent of age, sex, underlying cardiac pathology, duration since operation, drug treatment, and biopsy results.

\section{CHANGES IN HEART RATE}

\section{With posture}

Eight of the nine recipient hearts showed an increase in heart rate (by 7(2) beats/min) on standing up compared with 0 of nine donor hearts $(p<0 \cdot 01)$.

\section{During exercise}

Exercise was stopped in all patients because of fatigue at $7 \cdot 4(0.9)$ minutes (range 3-10 minutes). The increase in heart rate during the first three minutes of exercise was lower in the donor heart $(10.7(3.6)$ beats/min) than in the recipient heart $(30(4.8)$ beats/ min; $p<0.001$ ) (Fig. 2b). The magnitude of increase in the two heart rates showed, however, a significant correlation during this period $(r=0.75 ; p<0.05)$ (Fig. $3 b)$. During the later stages of exercise, heart rate increases in the donor heart were either similar to, or greater than, those in the recipient heart so that at peak exercise the two heart rates were not significantly different in the group as a whole $(147(3.3)$ beats $/ \mathrm{min}$ in donor hearts compared with $152(2.8)$ beats $/ \mathrm{min}$ in recipient hearts) (Fig. 2a). In addition, the two heart rates at peak exercise showed a significant correlation $(r=0.81 ; p<0.01)$ (Fig.3c).

\section{After cessation of exercise}

Five of the nine donor hearts but none of the recipient hearts showed an increase in heart rate after cessation of exercise $(p<0.05)$. The donor heart rate increased by $10.8(2)$ beats/min in these five patients, and a further two patients maintained peak heart rates for at least three minutes after exercise was stopped. The heart rate was equal to, or greater than, that at peak exercise for $3.2(0.7)$ minutes in the nine donor hearts during recovery. This immediate postexercise tachycardia was most prominent in magnitude and duration in the three patients exercised within three months of operation $(+7,+13$, and +16 beats/min; 4 minutes, 5 minutes, and 6 minutes respectively). The number of patients studied is, however, too small for statistical comparison. At three minutes after exer- cise, heart rate had decreased by only $0.2(5.7)$ beats/ $\mathrm{min}$ in the donor hearts compared with $33.6(6)$ beats/min in the recipient hearts $(\mathrm{p}<0.001)$ (Fig. 2c). Despite this there was a good correlation in the changes in heart rate between the two hearts $(r=0.83$; $\mathrm{p}<0.01$ ), as patients showing an increase in donor heart rate after exercise showed the smallest decrease in recipient heart rate measured over the same period (Fig. 3d).

The subsequent rate of decrease in the two heart rates was similar $(-12.8(1.5)$ beats/min in donor hearts compared with $-12(2.7)$ beats/min in the recipient hearts at 3-6 minutes after exercise) (Fig. 2c).

There was a weak trend when the magnitude of the heart rate decrease in the two hearts during this period was compared $(r=0.62 ; p<0.05)$.

Figure 4 illustrates the changes in rate in the donor and recipient hearts in a typical patient studied at four weeks and shows the increase in donor heart rate after cessation of exercise.

\section{RELATION OF RESTING RATE AND RECOVERY \\ RATE}

The resting heart rate did not correlate with the changes in the first three minutes of exercise or recovery in the donor hearts but showed significant inverse correlations in the innervated recipient hearts $(\mathrm{r}=0.85 ; \mathrm{p}<0.01$ and $\mathrm{r}=0.81 ; \mathrm{p}<0.01$ respectively). The increase in heart rate during the first three minutes of exercise correlated inversely with the decrease in heart rate during the first three minutes of recovery in both the donor $(r=0.79 ; p<0.01)$ and recipient hearts $(r=0.76 ; p<0.01)$ separately and overall $(r=0.87 ; p<0.001)$.

\section{COMPARISONS BETWEEN RECIPIENT HEARTS AND} CONTROL SUBJECTS

The resting heart rate in the heterotopic recipient hearts $(95(6)$ beats $/ \mathrm{min}$ ) was significantly greater than in the controls $(76(2)$ beats $/ \mathrm{min}) \quad(\mathrm{p}<0.001)$. Nevertheless, the increases in heart rate on standing (7(2) beats/min in the heterotopic recipient heart and $10(4)$ beats/min in the control subjects) and during the first three minutes of exercise (30(4) and 29(3) beats/ min respectively) were similar. The control subjects exercised longer, however, and reached higher heart rates (14(1) minute and $183(9)$ beats/min respectively) than the transplant patients $(8(2) \mathrm{min}$ and $152(2.8)$ beats/min; $p<0.01$ and 0.001 respectively). The decrease in heart rate in the first three minutes after the cessation of exercise was similar (expressed as a percentage of the total heart rate increase) in both the controls and recipient hearts $(-62(3) \%$ and $-57(8) \%)$. 

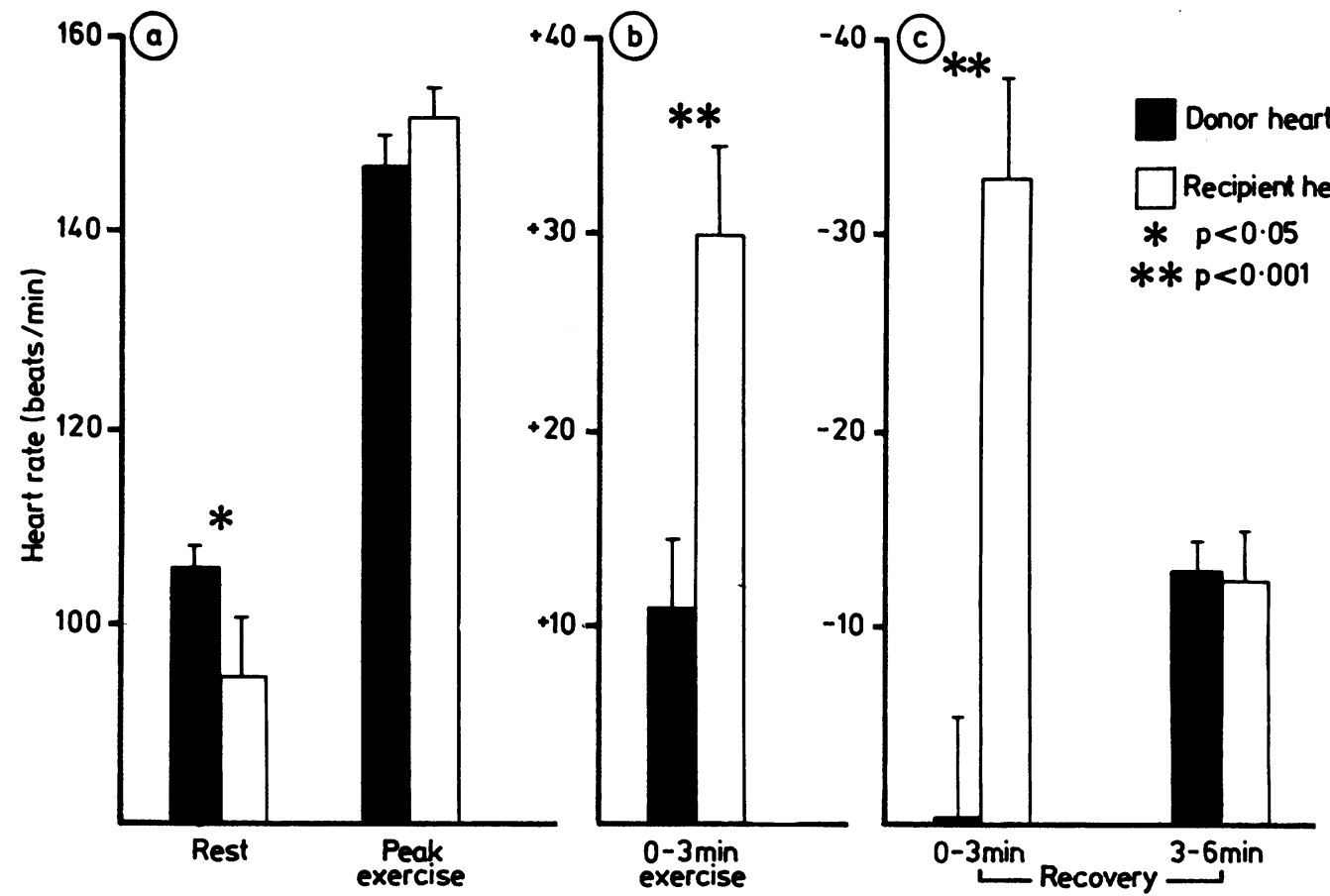

Fig. 2 Heart rates (a) at rest and at peak exercise, (b) in the first three minutes of exercise (\% increase), and (c) at recovery (\% decrease) in the donor and recipient hearts.

\section{Discussion}

The interrelation of the changes in the rate of the denervated and innervated hearts, in the same subject during identical stimuli, is a useful model to study the contribution of the nervous system to heart rate control since current evidence suggests that the donor heart remains denervated after transplantation, ${ }^{5}$ and the nerves to the recipient heart are not severed during heterotopic transplantation. Although this is the main factor that can explain the differences in response between the two hearts, other possible factors include the possible inhibitory influence of baroreceptor mediated stimuli (although these are of less importance during exercise ${ }^{6}$ ) on the recipient heart due to the added random ejection by the donor heart. In addition, the position of the heterotopic donor heart, in the right side of the chest, and the peculiar mode of atrial filling and aortic emptying produced by the specific type of anastomosis may influence its response. Although the recipient heart may have intact nerves and a patent arterial supply, the response to exercise can be modified by the underlying cardiac pathology (congestive cardiomyopathy or ischaemic heart disease). Comparison of the responses of the recipient heart with those of healthy controls suggests, however, similarity in the changes in heart rate response to standing during the first three minutes of exercise and recovery, although the resting heart rate, duration of exercise, and peak heart rate were significantly different. This suggests that it would be valid to compare the heart rate changes in the donor heart with those in the recipient heart of the same patient.

The resting heart rate of the donor heart was higher than that of the corresponding recipient heart in eight of nine patients, and this finding is consistent with vagal and sympathetic denervation. ${ }^{7}$ The heart rate of the heterotopic donor hearts that we observed is comparable to those found after pharmocological autonomic blockade, ${ }^{7}$ and is similar to the heart rate in patients with orthotopic human transplants in our own experience and that reported from other centres. ${ }^{28}$ The resting heart rate in the innervated recipient heart was inversely related to heart rate changes in the early minutes of exercise and recovery. This indicates that, with higher vagal tone, resting heart rate is lower and rises rapidly during early exercise owing to vagal withdrawal and falls rapidly after exercise owing to vagal stimulation. The resting heart rate in the recipient hearts was, however, significantly higher than in the controls and is probably related to 

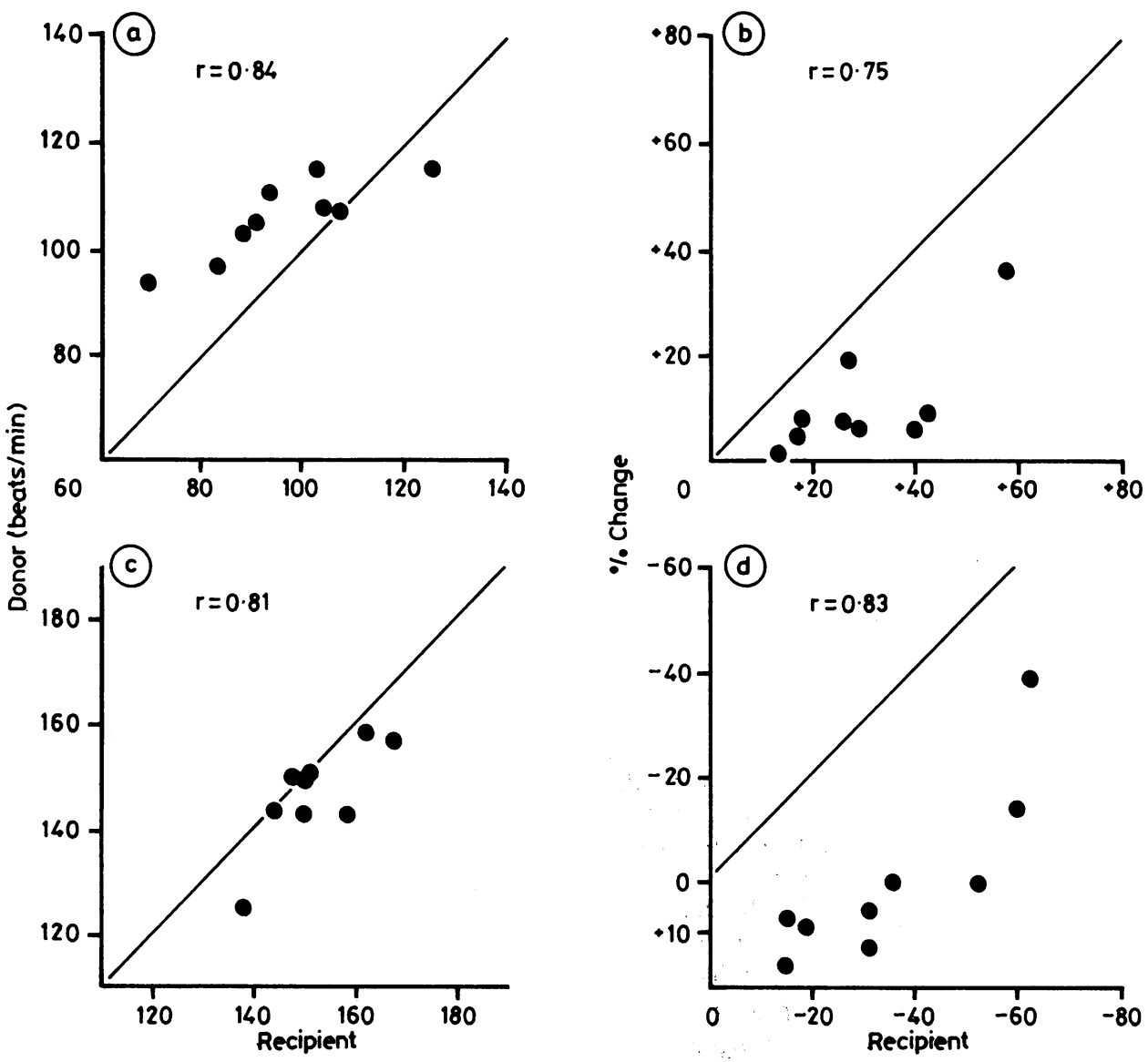

Fig. 3 Correlation between the increase in donor and recipient heart rate (a) at rest, (b) at 0-3 min exercise, (c) at peak exercise, and (d) at 0-3 min of recovery.

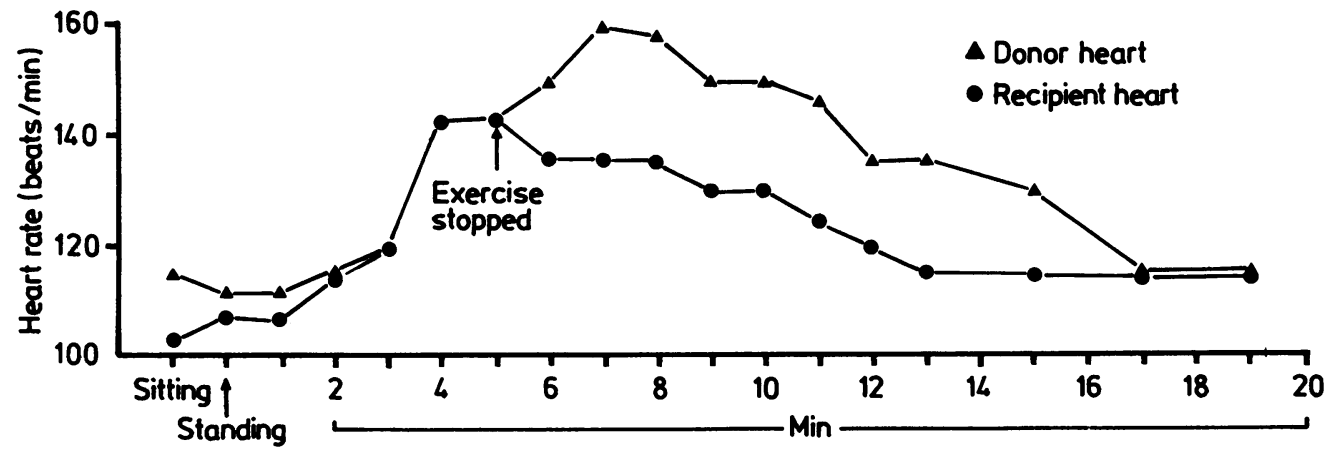

Fig. 4 Changes in heart rate in a patient at rest, on standing, and during and after exercise in the donor and recipient hearts. Note the similar heart rate at peak exercise and increase in heart rate after exercise in the donor heart. 
the fact that these are severely diseased hearts. A lack of immediate increase in heart rate on standing up in the donor hearts is consistent with denervation, ${ }^{9}$ and contrasts with the increase seen in the innervated recipient hearts.

Previous experimental work has shown that the autotransplanted canine heart is capable of normal work capacity. ${ }^{210}$ There are, however, pronounced differences in the initial response to exercise between the denervated and normal canine heart. An increase in heart rate predominates in the normally innervated heart, ${ }^{11}$ whereas increases in stroke volume predominate in the denervated heart ${ }^{12}$ with little initial heart rate increase. ${ }^{213}$ We have seen only a small increase in heart rate in the initial three minutes of exercise in the denervated donor heart whereas the corresponding increase in the innervated recipient heart was threefold. This slow initial acceleration of the donor heart ${ }^{14}$ is consistent with the reports of Pope et al in human orthotopic transplants. ${ }^{2}$ In addition, we have observed that the magnitude of initial heart rate increase in the innervated and denervated hearts correlated, suggesting that even at this low level of exercise there may be some chronotropic mechanisms that are common to the two hearts. These may be due either to low concentrations of circulating catecholamines or to some undefined intrinsic regulation in response to increased venous return.

During the latter phases of exercise the heart rate increase in the donor and recipient hearts was variable, but in general the two heart rates tended to converge so that at peak exercise the two heart rates were similar. This is of particular interest and suggests that at peak exercise non-neural mechanisms-for example, plasma catecholamines, which are at their maximum at peak exercise ${ }^{14}{ }^{15}$ - may be the chief mechanism and that any upregulation in $\beta$ receptor density ${ }^{16}$ and affinity in the denervated heart may almost exactly compensate for the absence of sympathetic nerve stimulation.

The greatest dissociation in the heart rate response of the denervated donor heart and innervated recipient heart was observed after the cessation of exercise. While the innervated heart rate decreased rapidly, the denervated hearts showed little change overall. Indeed, the heart rates were increased above those observed at peak exercise in five donor hearts for several minutes. These changes have not been reported before, either in experimental denervated hearts or after orthotopic transplantation. This may be due to the fact that in previous reports of exercise in orthotopic transplantation the studies were performed at 12-61 months ${ }^{2}$ or at 7-18 months. ${ }^{8}$ By contrast, our patients were exercised much earlier (1-6 months). The postexercise tachycardia was most pronounced in the three patients exercised under three months and may be a feature of the early months after cardiac transplantation. The mechanism(s) of these changes is unknown but may be due to the heightened response of the donor heart to the circulating catecholamines, which have been shown to continue to increase after exercise has stopped. ${ }^{17}$ In spite of the pronounced difference in heart rate changes between the two hearts during the first three minutes of recovery, there is a close correlation in individual patients suggesting the presence of non-neural linked mechanisms affecting both hearts.

This study has served to identify the specific interrelations between the heart rate at rest and the response during different phases of dynamic exercise. We hope that this will be of value in understanding the physiological mechanisms governing heart rate changes and help in the management of patients after cardiac transplantation or those with automatic nervous disorders.

\section{References}

1 Donald DE, Shepherd JT. Response to exercise in dogs with cardiac denervation. Am f Physiol 1963; 205: 393-400.

2 Pope SE, Stinson EB, Daughters GT II, Schroeder JS, Ingels NB $\mathrm{Jr}$, Alderman EL. Exercise reponse of the denervated heart in long-term cardiac transplant recipients. Am $\mathcal{f}$ Cardiol 1980; 46: 213-8.

3 Novitzky D, Cooper DKC, Barnard CN. The surgical technique of heterotopic heart transplantation. Am Thorac Surg 1983; 36: 476-82.

4 Bruce RA. Exercise testing of patients with coronary heart disease: principles and normal standards for evaluation. Ann Clin Res 1971; 3: 323-32.

5 Mason JW, Harrison DC. Electrophysiology and electropharmacology of the transplanted human heart. In Narula OS, ed. Cardiac arthythmias: electrophysiology, diagnosis and management. Baltimore: Williams and Wilkins, 1979: 66-81.

6 Bristow JD, Brown EB Jr, Cunningham DJC, et al. Effect of bicycling on the baroreflex regulation of pulse interval. Circ Res 1971; 28: 582-92.

7 Jose AD. Effect of combined sympathetic and parasympathetic blockade on heart rate and cardiac function in man. Am $\mathcal{f}$ Cardiol 1966; 18: 476-8.

8 Bexton RS, Milne JR, Cory-Pearce R, English TAH, Camm AJ. Effect of betablockade on exercise response after cardiac transplantation. Br Heart f 1983; 49: 584-8.

9 Smith OA. Reflex and central mechanisms involved in the control of the heart and circulation. Ammu Rev Physiol 1974; 36: 93-123.

10 Donald DE, Shepherd JT. Sustained capacity for exercise in dogs after complete cardiac denervation. Am $\mathcal{F}$ Cardiol 1964; 14: 8539.

11 Vatner SF, Franklin D, Higgins CB, Patrick T, Braunwald E. Left ventricular response to severe exertion in untethered dogs. $f$ Clin Invest 1972; 51: 3052-60.

12 Stinson EB, Griepp RB, Schroeder JS, Dong E Jr, Shumway NE. Hemodynamic observations one and two years after cardiac transplantation in man. Circulation 1972; 45: 1183-94.

13 Hallman GL, Leatherman LL, Leachman RD, et al. Function of the transplanted human heart. F Thorac Cardiovasc Sung 1969; 58: 318-25.

14 Warren JB, Dalton N, Turner C, Clark TJH, Toseland PA. Adrenaline secretion during exercise. Clin Sci 1984; 66: 87-90.

15 Christensen NJ. Sympathetic nervous activity during exercise. Arrue Rev Physiol 1983; 45: 139-53.

16 Lurie KG, Bristow MR, Reitz BA. Increased B-adrenergic receptor density in an experimental model of cardiac transplantation. $f$ Thorac Cardioevasc Sung 1983; 86: 195-201.

17 Dimsdale JE, Hartley LH, Guiney T, Ruskin JN, Greenblatt D. Postexercise peril, plasma catecholamines and exercise. $\mathcal{F} A M A$ 1984; 251: 630-2. 